\title{
Observation of narrow Autler-Townes components in the resonant response of a dense atomic gas
}

\author{
Vladimir A. Sautenkov, ${ }^{1,2}$ Yuri V. Rostovtsev, ${ }^{1}$ and Eric R. Eliel $^{3}$ \\ 1 Department of Physics and Institute of Quantum Studies, \\ Texas AEM University, College Station, Texas 77843-4242 \\ 2 P. N. Lebedev Institute of Physics, 119991 Moscow, Russia \\ ${ }^{3}$ Huygens Laboratory, Leiden University, P.O. Box 9504, 2300 RA Leiden, The Netherlands
}

(Dated: October 25, 2018)

\begin{abstract}
We have experimentally studied the reflection of a weak probe beam from a dense atomic potassium vapor in the presence of a strong laser field tuned to the atomic resonance transition. We have observed an Autler-Townes doublet under hitherto unexplored conditions, namely that the Rabi frequency induced by the strong laser field is much smaller than the self-broadened width of the resonance transition of the unexcited vapor. We attribute our observation to a reduction of the atomic decoherence by the strong drive field. We present a theoretical model of nonlinear processes in a dense atomic gas to explain the observed results.
\end{abstract}

PACS numbers: PACS numbers: 32.70.-n, 42.50.Hz, 42.50.Nn

Spectral line broadening is a universal phenomenon and a multitude of techniques has been devised to reduce or eliminate it altogether, enabling the study of spectral features that would, otherwise, remain hidden [1, 2, 3, 4]. In particular this applies to atomic or molecular gases where the collision rate can be made sufficiently small by, for instance, rarefying the vapor. However, when the interest lies with the (strongly) interacting gas, line broadening becomes the essence, and the study of the width of the fundamental resonance transitions in highdensity atomic vapors is well-documented [5, 6].
(A)

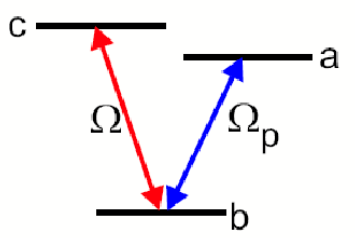

(C)

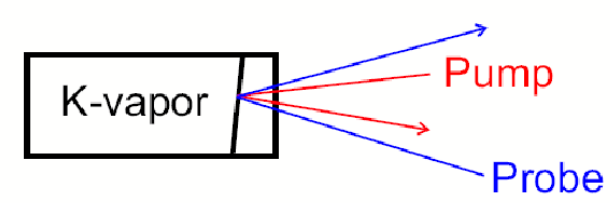

FIG. 1: (Color online) Energy-level scheme for potassium. The bare atomic states are shown in (A), where level $a$ corresponds to the $4 p^{2} P_{1 / 2}$ excited state, level $b$ to the $4 s^{2} S_{1 / 2}$ ground state, and level $c$ to the $4 p^{2} P_{3 / 2}$ excited state. The strong drive field, with Rabi frequency $\Omega$, couples states $b$ and $c$, while the probe laser beam, with Rabi frequency $\Omega_{\mathrm{p}}$ is scanned over the $b-a$ transition. Frame (B) shows the probe transition in the dressed basis, the states being written as $|+\rangle$ and $|-\rangle$, and (C) provides a sketch of the experimental setup.
Although, in the general case, the broadening of the resonance line has many contributions [7], for dense homogeneous gases, the major contribution is caused by the resonant dipole-dipole interaction between ground- and excited-state atoms of the same species, and is known as self- or resonance broadening [5, 6]. The interaction is long range, so that already at reasonably modest densities of order $10^{17} \mathrm{~cm}^{-3}$ one leaves the binarycollision regime and multi-perturber effects may come into play [6, 8]. It has, for instance, been shown that the Zeeman effect is modified at atomic densities of this order [9]. We note that multi-perturber effects were observed in high presure buffer gas [10, 11, 12] when the duration of collisions cannot be neglected.

In a high-density vapor the atom responds not just to the externally applied EM field but also to the field reradiated by other atoms in the vapor, i.e. it responds to the local field. As first shown by Lorentz, this causes the position of the resonance to be shifted [13]. During the last decade this local-field shift, named after Lorentz, has been studied by both frequency [14, 15, 16, 17, 18, 19] and time-domain 20 techniques. It is proportional to the atomic density, and of the same order of magnitude as the line width [21].

It has been predicted that the Lorentz local-field shift depends on the degree of (incoherent) excitation of the vapor [21, 22], a prediction that stimulated the study of a variety of nonlinear optical phenomena in dense atomic vapors, such as piezophotonic switching and lasing without inversion [23]. The experimental demonstration of the excitation dependence of the Lorentz shift [17, 19] brought to light that the self-broadened width itself is also excitation dependent, an effect that could be explained in terms of a quasistatic (multi-perterber, quasimolecular) collision model [6, 17]. Recently, it was shown by using a time-domain technique, that non-Markovian collision dynamics can be observed in these high density 
resonantly-broadened vapors, indicating that the duration of a collision can no longer be neglected [24].

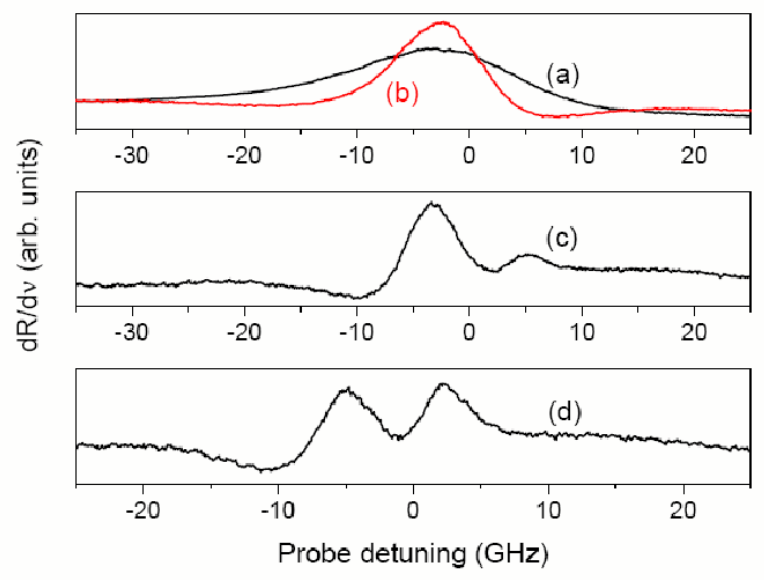

FIG. 2: (Color online) Frequency-modulated reflectivity spectra of a potassium vapor at a density of $4.9 \times 10^{17} \mathrm{~cm}^{-3}$, as measured on the $\mathrm{D}_{1}$ transition. Curves(a) and (b) display the FM reflection spectrum of the vapor when the pump laser is switched off, and when it operates at a power of $\approx 0.5 \mathrm{~W}$ and is tuned far $(130 \mathrm{GHz})$ above the $\mathrm{D}_{2}$ transition. When the detuning of the pump laser is reduced to $\approx 3 \mathrm{GHz}$ the spectral line in the FM spectrum becomes an Autler-Townes doublet (curve (c)). Curve (d) shows the Autler-Townes components at zero pump detuning.

The experimental study of resonance broadening remains a topical issue in view of theoretical claims that, because of the long-range nature of the interaction, many-body effects play a role at all densities [6]. Here we show that at a higher number density of $4.9 \times 10^{17} \mathrm{~cm}^{-3}$ the atomic ensemble can be made to evolve coherently as if it is a two-level system with intensity dependent line width. We deduce this behavior from our measurements of the Autler-Townes splitting [25] of the potassium atomic ground state. Observation of the AutlerTownes splitting of optical transition in delute gases were previously reported in [26]. Essential to our observation is the effect of collision-induced line narrowing [16, 17, 19].

At the number density of our experiment, the selfbroadened line width of both resonance lines of potassium is considerably larger than either their hyperfine structure or their Doppler width. Under these conditions the level structure of potassium is well-represented by a three-level scheme, shown in Fig. 1. A strong driving field (Rabi frequency $\Omega$ ) couples levels $b$ and $c$, while a weak probe field, with Rabi frequency $\Omega_{\mathrm{p}}$ is scanned over the $a \leftrightarrow b$ transition.

The experiment is performed in reflection (see Fig. 11C), using an experimental arrangement similar to that of Ref. [19]. The strong coupling field is produced by a CW Ti- sapphire laser tuned close to the $D_{2}$-line $(\lambda=766 \mathrm{~nm})$ of atomic potassium. The laser beam, having a maximum power of $\approx 0.5 \mathrm{~W}$, has its focus (the area of the beam is $510^{-4} \mathrm{~cm}^{2}$ ) at the window of a vapor cell that is constructed entirely out of sapphire. A weak probe beam from an external cavity diode laser is focussed to overlap with the pump beam at the entrance window of the cell. The probe laser is scanned over the $D_{1}$-line at $\lambda=770 \mathrm{~nm}$, at a power level of $\approx 10^{-4} \mathrm{~W}$, far below the onset of saturation (saturation parameter [3] is $<0.1$ ). Both the pump and probe beams are linearly polarized and are incident on the sapphire cell at near-normal incidence. While being scanned, the probe laser is frequency modulated over a range of $100 \mathrm{MHz}$ at a frequency of 400 $\mathrm{Hz}$. The reflected probe beam is captured by a photodiode whose signal output is fed to a lock-in amplifier. Our experiment runs at an atomic density of $4.9 \times 10^{17} \mathrm{~cm}^{-3}$, yielding a self-broadened linewidth $\Gamma_{\text {self }} / 2 \pi$ of $28.4 \mathrm{GHz}$ for the $\mathrm{D}_{1}$ line $[9]$.

Figure 2 shows the measured FM-modulated spectra. The zero on the frequency axis refers to the center frequency of the absorption spectrum of a low density $\left(N \simeq 10^{11} \mathrm{~cm}^{-3}\right)$ potassium vapor in a reference glass cell. Curve (a) displays the reflection spectrum when the pump beam is switched off, i.e. it shows the FM linear reflectivity of the sapphire/high-density vapor interface. When the pump laser is switched on, operates at an output level of $0.5 \mathrm{~W}$, and is tuned $130 \mathrm{GHz}$ above the $\mathrm{D}_{2}$ line, the FM spectrum of curve (b) is obtained. At this large detuning the pump-laser beam penetrates a considerable distance into the vapor cell. Due to radiation trapping the decay of the excited-state population is slowed down and, as a result of radiative transport, the vapor near the sapphire window becomes homogeneously and incoherently excited. In line with earlier observations ([19]) one notices that the FM spectrum of the strongly excited vapor (curve (b)) is narrower than its unexcited counterpart (curve (a)), and that their centers do not coincide. However, both spectra are described by the same spectral function, with adjusted values for the Lorentz local-field shift and self-broadened width [16, 17, 19].

The spectral width $\Gamma_{\text {self }}$ of the resonance line can be estimated from the interval $\Delta \omega_{\mathrm{mm}}$ between the maximum and minimum of the reflection spectrum or, equivalently, from the zeros of the FM spectrum. For the $D_{1}$ transition one has $\Gamma_{\text {self }}=0.87 \Delta \omega_{\mathrm{mm}}[9,19]$. By this method, we determine the line width for the unexcited vapor as 31 $\mathrm{GHz}$ (Fig. 22a), in reasonable agreement with the value $\Gamma_{\text {self }} / 2 \pi=28.4 \mathrm{GHz}$, calculated using the known selfbroadening coefficient and our estimate for the atomic density. Similarly, we estimate the line width for the incoherently excited vapor as just $11 \mathrm{GHz}$ (Fig. 2b). Hereby we show that the excitation dependence of the self-broadened line width is a valid concept also at the vapor density of the present experiment.

In order to observe coherent effects we tune the pump 
laser close to the center of the atomic line. Curves (c) and (d) show the experimental FM reflectivity spectra for a pump detuning of 3 and $0 \mathrm{GHz}$, respectively. In both cases one observes that the single resonance of curve (b) is split into an Autler-Townes doublet [25, 26]. The asymmetry of the doublet in curve (c) reflects the fact that, for this curve, the pump laser is tuned slightly above the $\mathrm{D}_{2}$ resonance transition, while the near-perfect symmetry of curve (d) shows that the pump laser is, in that case, tuned on resonance. Note that, as a result of the various line shifts, there is no easy method, beyond looking at the symmetry of the Autler-Townes doublet, to establish whether the pump laser is tuned exactly to resonance or not.

To obtain a good estimate for the width and separation of the the components of the Autler-Townes doublet we have made a non-linear fit of the experimental data of curve (d) in Fig. 2, yielding a width of $8.5 \mathrm{GHz}$ and a splitting of $7.6 \mathrm{GHz}$.

It is well known that the separation between the two components of the Autler-Townes doublet equals the Rabi frequency induced by the pump laser [3, 27]. For the conditions of the present exeriment where hyperfine and Zeeman substructure can be ignored, the Rabi frequency $\Omega$ for the potassium $D_{2}$ line can be expressed as

$$
\Omega / 2 \pi=8 \times 10^{7} \sqrt{I\left[\frac{\mathrm{W}}{\mathrm{cm}^{2}}\right]} \mathrm{Hz},
$$

where $I=c E^{2} /(8 \pi)$ is the pump laser intensity (in units of $\left.\mathrm{W} / \mathrm{cm}^{2}\right)$. Here the Rabi frequency is written as

$$
\Omega=E \wp_{x} / \hbar,
$$

with $E$ the optical electric field and $\wp_{x}$ the projection of the atomic dipole moment $\vec{\wp}$ along that field. The value of $\wp_{x}=\wp / 3$ has been obtained from experimental data for the spontaneous lifetime [28]. Using Eq. (11) we calculate the Autler-Townes splitting to be $8 \mathrm{GHz}$ at a pump power of $0.5 \mathrm{~W}$, in satisfying agreement with the experimental value $(7.6 \mathrm{GHz})$.

We have recorded the Autler-Townes doublet for several values of the detuning $\Delta$ and intensity of the pump laser. When $|\Delta|$ increases one of the components of the doublet becomes stronger while the other fades away. When $|\Delta / 2 \pi|>6 \mathrm{GHz}$ the weak component can no longer be measured, and only a single resonance is observed. For this case, where the pump laser is detuned from exact resonance, the Autler-Townes splitting is given by the generalized Rabi frequency [27]

$$
\tilde{\Omega}=\sqrt{\Omega^{2}+\Delta^{2}} .
$$

The measured values of the Autler-Townes splitting as a function of the generalized Rabi frequency $\tilde{\Omega}$ are presented in Fig. 3. The three points for $\tilde{\Omega} / 2 \pi \leq 8 \mathrm{GHz}$ have been measured at zero detuning and pump laser powers equal to $0.3,0.4$ and $0.5 \mathrm{~W}$, respectively. The points at $\tilde{\Omega} / 2 \pi \geq 8.5 \mathrm{GHz}$ were measured by using a pump power equal to $0.5 \mathrm{~W}$ and detunings of $\Delta / 2 \pi= \pm 3 \mathrm{GHz}$ and \pm 6 $\mathrm{GHz}$, respectively. Also shown is a linear fit through the data; its slope equals 0.92 , in quite good agreement with the expected value, equal to 1 (the error of slope is $2 \%$ and the difference between theoretical and experimental curves is about $6 \%$ due to systematic uncertanties).

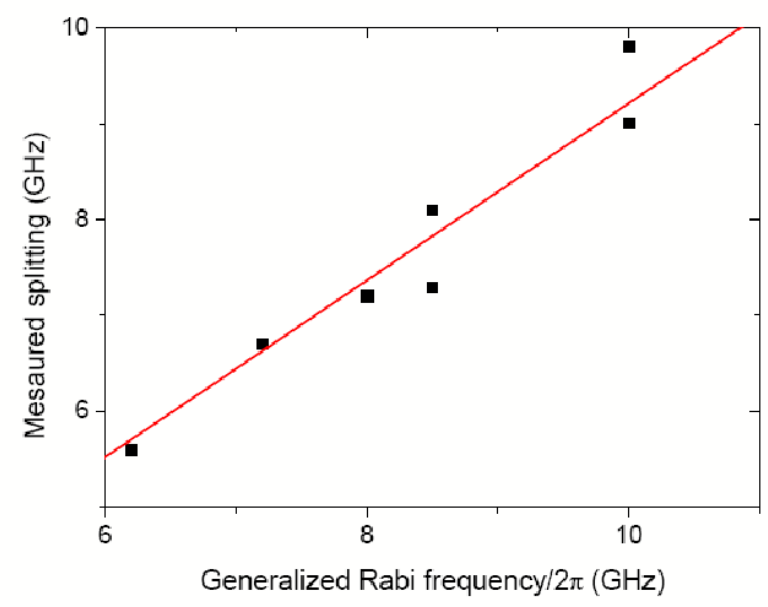

FIG. 3: The measured Autler-Townes splitting versus generalized Rabi frequency. The solid line shows the result of a linear fit.

To explain our results we use the optical Bloch equations for a V-type three-level atom, suitably modified for the fact that the system under study is a dense rather than a dilute vapor. From these equations we evaluate the susceptibility of the probe at frequency $\omega_{p}$ as 29]

$$
\chi\left(\omega_{p}\right)=i \frac{3 \lambda^{2} \gamma}{8 \pi} \frac{\left(n_{a}-n_{b}\right)+\frac{\Omega^{2}}{\Gamma_{c b} \Gamma_{c a}}\left(n_{c}-n_{b}\right)}{\Gamma_{a b}+\frac{\Omega^{2}}{\Gamma_{c a}}} .
$$

Here the level populations $n_{a}, n_{b}$ and $n_{c}$ depend on the Rabi frequency of the driving field $\Omega, \gamma$ is the radiative rate on the $a \rightarrow b$ transition, and the generalized coherence decay rates are $\Gamma_{c b}=\tilde{\gamma}_{c b}+i \delta_{c b}, \Gamma_{c a}=\tilde{\gamma}_{c a}+i \delta_{c a}$, $\Gamma_{a b}=\tilde{\gamma}_{a b}+i \delta$. The frequency of the pump is denoted as $\bar{\omega}$, and its detuning is given by $\delta_{c b}=\omega_{c b}-\bar{\omega}+\Delta \omega_{L}+\Delta \omega_{c}$, with $\Delta \omega_{L}$ the Lorentz local field shift, and $\Delta \omega_{c}$ the collisional frequency shift, which is independent of the level of excitation [14, 15, 19]. The detuning of the probe from resonance is $\delta=\omega_{a b}-\omega_{p}+\Delta \omega_{L}+\Delta \omega_{c}$, with $\omega_{p}$ the frequency of the probe laser. For the two photon detuning we write $\delta_{c a}=\omega_{c a}-\bar{\omega}+\omega_{p}+\Delta \omega_{L}+\Delta \omega_{c}$. For $\tilde{\gamma}_{c b}, \tilde{\gamma}_{c a}$ and $\tilde{\gamma}_{a b}$ we write $\tilde{\gamma}_{a b}=\gamma / 2+\Gamma_{\text {self }} \simeq \Gamma_{\text {self }}$, $\tilde{\gamma}_{c b}=\gamma / 2+\Gamma_{\text {self }} \simeq \Gamma_{\text {self }}$, and $\tilde{\gamma}_{c a}=\gamma+\Gamma_{\text {self }} \simeq \Gamma_{\text {self }}$, since the self-broadened line width is much larger than the natural width of the transitions. 
For the self-broadened width we write [17, 19]

$$
\Gamma_{\text {self }}=k n_{b},
$$

with $k$ the self-broadening coefficient [5]. The Lorentz local-field shift is given by [21, 22]

$$
\Delta \omega_{L}=\frac{k}{3}\left(n_{b}-n_{a}\right)
$$

The reflectivity of the coherently driven dense vapor can now be evaluated using

$$
R(\omega)=\left|\frac{n(\omega)-n_{0}}{n(\omega)+n_{0}}\right|^{2},
$$

with $n(\omega)=\sqrt{1+4 \pi \chi(\omega)}$ and $n_{0}$ the refractive indices of the vapor and of the window material of the vapor cell, respectively. Using Eq. (7) and appropriate values for the experimental parameters we calculate the FM reflection spectra, as shown in Fig. 4, for a pump detuning of zero and $3 \mathrm{GHz}$, respectively, and a pump Rabi frequency of $12 \mathrm{GHz}$. Fully in line with the experimental result our theoretical model predicts a well-resolved Autler-Townes doublet under circumstances that the Rabi frequency is much smaller than the unsaturated self-broadened line width.
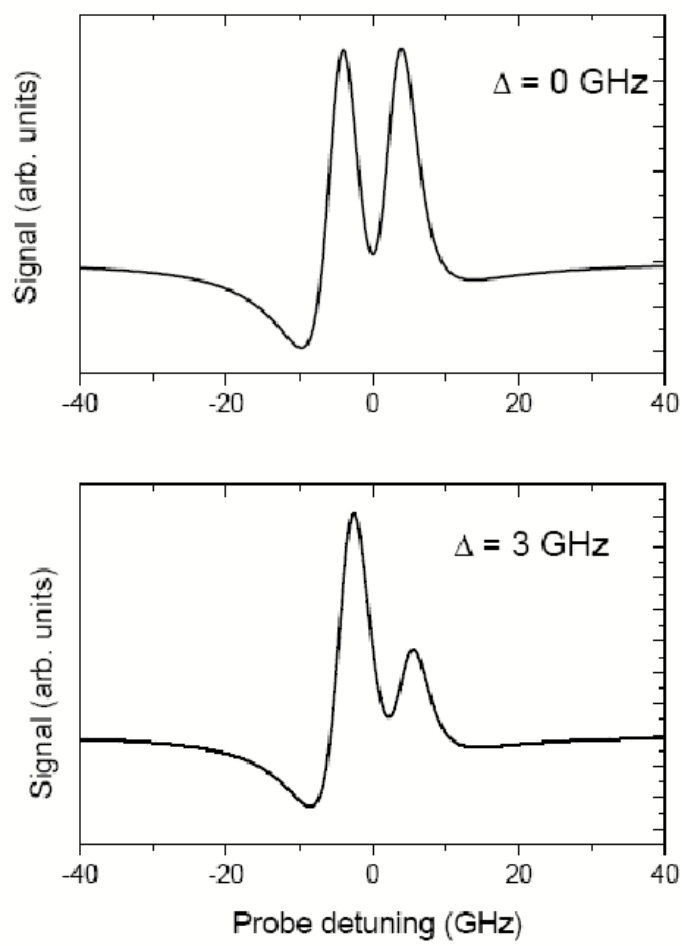

FIG. 4: Results of the theoretical model for the FM-reflection spectrum of a vapor of coherently driven V-type three-level atoms at high density, using Eqns. (4) and (7).
It is worth to mention here that, even though the experiments and theoretical analysis have been done for conditions corresponding to hot dense gases, it will be interesting to study discussed multy-perturber effects in dense cold atomic and molecular gases, and possible influence on coherent backscattering in cold atoms [30].

In conclusion, we have observed the Autler-Townes splitting of the atomic ground state in the reflection spectrum of a dense potassium vapor in a regime where the separation of the two Autler-Townes components, as given by the Rabi frequency, is much smaller than the nominal self-broadened width of the transition. We attribute our observation to the fact that the strong drive field, in addition to inducing coherent evolution of the atomic levels, also causes a dramatic narrowing of the spectral line. Taking into account the excitation dependence of both the local-field shift and the resonance line width we obtain good agreement between our experimental results and an effective three-level model based on modified Bloch equations.

We wish to acknowledge S.T. Cundiff, H. van Kampen, S. Mukamel, and J. P. Woerdman for useful discussions and beneficial help. This work was done, in part, while V.A.S. was a visitor at Leiden University as part of the research program of the "Stichting voor Fundamental Onderzoek der Materie". V.A.S. and Yu.V.R. also gratefully acknowledge the support from the NSF, the Office of Naval Research, the Air Force Research Laboratory (Rome, NY), Defense Advanced Research Projects Agency, and the Robert A. Welch Foundation (Grant \#A-1261).

[1] D. Meschede, Optics, Light and Lasers, (Wiley-VCH, Weinheim, Germany, 2004).

[2] M. D. Levenson and S. S. Kano, Introduction to nonlinear laser spectroscopy, (Academic Press, San Diego, 1988).

[3] Robert W. Boyd, Nonlinear Optics (San Diego, CA : Academic Press, 2003).

[4] S. Svanberg, Atomic and Molecular Spectroscopy, (Springer, Berlin, 1992).

[5] E. L. Lewis, Phys. Rep. 58, 1 (1980).

[6] J. A. Leegwater and S. Mukamel, Phys. Rev. A 49, 146 (1994).

[7] I.I. Sobelman, L.A. Vainshtein, E.A. Yukov Excitation of atoms and broadening of spectral lines (Berlin, New York, Springer-Verlag, 1981). Spectral line shapes : 18th International Conference on Spectral Line Shapes, Auburn, Alabama, 4-9 June 2006, Editors, Eugene Oks, Michael Pindzola (Melville, N.Y. : American Institute of Physics, 2006). E.A. Oks, Plasma spectroscopy : the influence of microwave and laser fields, (Berlin, New York, SpringerVerlag, 1995). Hans R. Griem, Spectral line broadening by plasmas (New York, Academic Press, 1974).

[8] W. P. West and A. Gallagher, Phys. Rev. A 17, 1431 (1978).

[9] H. van Kampen, A. V. Papoyan, V. A. Sautenkov, P. 
H. A. M. Castermans, E. R. Eliel, and J. P. Woerdman, Phys. Rev. A 56, 310 (1997).

[10] R. E. Walkup, A. Spielfiedel, and D. E. Pritchard, Phys. Rev. Lett. 45, 000986 (1980);

[11] J.P. Woerdman, E.J. Blok, M. Kristensen, et al., Phys. Rev. A 53, 1183 (1996).

[12] R.S. Trawinski, R. Ciurylo, J. Szudy, Phys. Rev. A74, 022716 (2006).

[13] H. A. Lorentz, Verh. Kon. Ned. Acad. Wet. 18, 1 (1879).

[14] J. J. Maki, M. S. Malcuit, J. E. Sipe, R. W. Boyd. Phys. Rev. Lett. 67, 972 (1991).

[15] J. J. Maki, W. V. Davis, R. W. Boyd, J. E. Sipe, Phys. Rev. A 46, 7155 (1992).

[16] H.Li, T.S. Varzhapetyan, V.A. Sautenkov, Yu.V. Rostovtsev, H. Chen, D. Sarkisyan, and M.O. Scully, accepted to be published in Appl.Phys. B (2008).

[17] V. A. Sautenkov, H. van Kampen, E. R. Eliel, and J. P. Woerdman, Phys. Rev. Lett. 77, 3327 (1996).

[18] P. Wang, A. Gallagher, and J. Cooper, Phys. Rev. A 56, 1598 (1997).

[19] H. van Kampen, V. A. Sautenkov, C. J. C. Smeets, E. R. Eliel and J.P. Woerdman, Phys. Rev. A 59, 271 (1999).

[20] S. T. Cundiff, Laser Physics, 12, 1073 (2002).

[21] R. Friedberg, S. R. Hartmann and J. T. Manassah, Phys. Rep. 7, 101 (1973); Phys. Rev. A 42, 5573 (1990).
[22] J. T. Manassah, Phys. Rep. 101, 359 (1983).

[23] A. S. Manka, J. P. Dowling, C. M. Bowden and M. Fleischhauer, Phys. Rev. Lett. 73, 1789, (1994).

[24] V. O. Lorenz and S. T. Cundiff, Phys. Rev. Lett. 95, 163601 (2005). V.O. Lorentz, S. Mukamel, W. Zhuang and S.T. Cundiff, Phys. Rev. Lett. 100, 013603 (2008).

[25] S. H. Autler, and C. H. Townes, Phys. Rev. 100, 703 (1955).

[26] A. Schabert, R. Keil, P.E. Toschek, Opt. Commun. 13, 265 (1975); A. Schabert, R. Keil, P.E. Toschek, Appl. Phys. 6, 181 (1975).

[27] C. Cohen-Tannoudji, J. Dupont-Roc, G. Grynberg, Atom-Photon Interactions: Basic Processes and Applications (Wiley, New-York, 1992) Chap.VI.

[28] I. I. Sobelman, Atomic Spectra and Radiative Transitions, (Springer, Berlin, 1992).

[29] M. O. Scully and M. S. Zubairy, Quantum Optics (Cambridge University Press, Cambridge 1997).

[30] D. Meiser, T. Miyakawa, H. Uys, P. Meystre, Advances in Atomic, Molecular, and Optical Physics 53, 151 (2006). G. Labeyrie, F. de Tomasi, J.C. Bernard, C.A. Muller, C. Miniatura, R. Kaiser, Phys. Rev. Lett. 83, 5266 (1999). D.V. Kupriyanov, I.M. Sokolov, C.I. Sukenik, M.D. Havey, Laser Phys. Lett. 3, 223 (2006). 